Published in final edited form as:

Conf Proc IEEE Eng Med Biol Soc. 2018 July ; 2018: 2264-2267. doi:10.1109/EMBC.2018.8512875.

\title{
Using Interictal HFOs to Improve the Identification of Epileptogenic Zones in Preparation for Epilepsy Surgery
}

\author{
Sina Farahmand [Student Member, IEEE], \\ Laboratory of Neural Engineering Research, Biomedical Engineering Department, Illinois Institute \\ of Technology (IIT), Chicago, IL, 60616-3793 USA, (sfarahma@hawk.iit.edu).
}

Tiwalade Sobayo [Member, IEEE], and Laboratory of Neural Engineering Research, Biomedical Engineering Department, Illinois Institute of Technology (IIT), Chicago, IL, 60616-3793 USA, (tsobayo@iit.edu).

\section{David J. Mogul [Senior Member, IEEE]}

Laboratory of Neural Engineering Research, Biomedical Engineering Department, Illinois Institute of Technology (IIT), Chicago, IL, 60616-3793 USA. He is also director of the Center for Integrative Neuroscience \& Neuroengineering Research, Illinois Institute of Technology, Chicago, IL, 60616-3793 USA, (Mogul@iit.edu)

\section{Abstract}

For the more than 15 million patients who have drug-resistant epilepsy, surgical resection of the region where seizure arise is often the only alternative therapy. However, the identification of this epileptogenic zone (EZ) is often imprecise. Generally, too little EZ identification and resection may cause seizures to continue and too much resection of it may lead to unnecessary neurological deficits. In this paper, an analytical methodology based on empirical mode decomposition (EMD) is proposed to improve the localization of the EZ for epilepsy patients. In this method, the instantaneous energy of interictal high frequency oscillations (HFOs), extracted from intracranial EEG (iEEG) recordings, are utilized as biomarkers for the EZ identification. The proposed method may significantly improve the precision by which pathological brain tissue is identified.

\section{Introduction}

Epilepsy is a chronic, neurological disease characterized by recurrent and spontaneous aberrant electrophysiological behavior in the brain, called epileptic seizures [1]. Epilepsy disease affects more than 50 million people worldwide. In approximately one-third of the cases, antiepileptic drugs fail to control seizures. Epilepsy surgery is a treatment option for these drug-resistant patients, in which an area of the brain causing the seizures is either resected or ablated.

The goal of resective surgery for intractable seizures is the removal of the epileptogenic zone (EZ) defined as the region of the brain that is responsible for generating seizures [2]. Theoretically, resection of the EZ should fully prevent future seizures. Pre-surgical evaluation is aimed at delineating the EZ which may, in some cases, arise from an epileptogenic lesion that is the presumed etiology of epileptic seizures. However, the EZ may be within the lesion or distant from it, or there may be no apparent lesion at all. The 
seizure onset zone ( $\mathrm{SOZ}$ ) is the area of the brain where clinical seizures originate and is thought to most closely approximate the EZ. However, even SOZ identification is restricted due to the ability to record intracranial electroencephalography (iEEG) from only a subset of the brain and the potential for rapid spread of seizure activity. In some cases, when the SOZ and an epileptogenic lesion are in agreement, the EZ can be easily identified. Precise identification of the EZ prior to resective surgery reduces the risk of damage to the eloquent cortex that could result in severe functional deficits. Surgical planning for patients to decide whether their SOZ includes eloquent cortex would greatly benefit from a more accurate approximation of the EZ. In the past, several studies have suggested that localized high frequency oscillations (HFOs) detected during iEEG recording are a way to spatially locate the EZ [3]-[7]. HFOs are characterized as spontaneous, non-stationary, low-amplitude electrophysiological activity with a frequency range between $80-500 \mathrm{~Hz}$ [8], [9]. Furthermore, HFOs are divided based on their spectral content into ripple $(80-250 \mathrm{~Hz})$ band and fast-ripple $(250-500 \mathrm{~Hz})$ band.

It should be noted that most algorithms for HFO analysis and detection have been based on visual inspection or made several assumptions regarding time-series, features of HFOs such as their number of oscillations, duration, and peak-to-peak amplitude. The main contributions of this paper are as follows. (1) Based on empirical mode decomposition (EMD) technique, we propose a data-driven, novel, and automated method for detecting clinically relevant HFO areas in iEEG recordings that eliminate the constraint of visual inspection. (2) We compare our detected HFO areas with the SOZ, as determined by epileptologists through detection of clear, ictal, iEEG discharges. (3) Our method can identify the EZ, which is related to the SOZ, using long periods of interictal iEEG signals without the need for precise timing of pre-ictal and ictal iEEG events.

The rest of the paper is organized as follows. Section II describes specifications of the adopted dataset along with the analytical methodology used for identification of the EZ. In section III, experimental results regarding the performance of using the proposed interictal HFO analysis to identify the EZ are presented. Finally, section IV provides conclusions to this research.

\section{Dataset and Methods}

\section{A. Dataset}

Samples of long-term, invasive, iEEG data recorded from two patients who subsequently underwent epilepsy surgery, at the Neurosurgery Department of the University Hospital of Zurich, Switzerland, were used in this study [10]. Recordings were made using subdural strip and grid electrodes as well as depth electrodes. From each night's recording, up to seven sample intervals, each containing five minutes of interictal slow-wave sleep were selected for our analysis. Intracranial data acquisition was performed using a Neuralynx system with a sampling frequency of $4000 \mathrm{~Hz}$, which was later downsampled to $2000 \mathrm{~Hz}$ for HFO analysis and $0.5-1000 \mathrm{~Hz}$ band-pass filtering. It should be noted that sampled-data from at least 3 hours distal from seizures was selected to eliminate the effect of ictal activity in our analysis. 
Additional clinical information regarding the two analyzed patients and data are provided in Table I. For each epilepsy patient, the channels covering the SOZ were assigned by epileptologists and were identified as the region most proximal to iEEG recording sites where ictal discharges were first observed. It should be noted that samples of iEEG data recorded from subdural grid electrodes in both patients were used in our HFO front-end analysis.

\section{B. Empirical Mode Decomposition (EMD)}

The iEEG data used in our analysis were recorded based on a common intracranial reference. Bipolar derivations from iEEG data were measured by taking the voltage difference between adjacent sites. This is to eliminate the confounding effects of common reference signal and volume conduction in our HFO detection [11]. Next, the bipolarderivate signals were decomposed into a series of intrinsic mode functions (IMFs) using the empirical mode decomposition method. EMD is an adaptive, non-linear method of decomposition that produces a set of finite nearly orthogonal oscillators that together comprise the basis functions within a dataset [12]-[14]. The basis functions are determined from the underlying dynamics of the signal itself, which may be non-linear and/or nonstationary.

\section{HFOs Detection}

Each iEEG time series was divided in consecutive, nonoverlapping, one-second windows. For each window, EMD was performed on the time series and the second and third IMFs were selected for further analysis. These two IMFs were chosen because their instantaneous frequencies always lie within the ripple and fast ripple bands. Fig. 1 exhibits the EMD process on a one-second bipolar-derivate iEEG signal. The second extracted IMF, which indicates HFO activity in the fast-ripple band, is illustrated in Fig. 1(b). Furthermore, the third IMF extracted from the one-second iEEG signal, which reflects HFO activity in the ripple band, is shown in the Fig. 1(c).

The Hilbert analytic signal was constructed for each IMF in order to calculate the instantaneous phase and amplitude. It should be noted that the instantaneous frequencies of the extracted IMFs are measured through taking derivatives of the instantaneous phases [15]. The energy of IMFs over one-second windows were measured as the mean of the square of the instantaneous amplitude. Using the whole five minutes for each interval, a threshold was determined for the energy of the second and third IMFs respectively through measuring the mean plus three times the standard deviation, $(\mu+3 \sigma)$. Electrode sites with energy-levels exceeding the threshold on both the second and third IMFs were analyzed for the HFO rates over the entire five-minute interval. Channels whose rate exceeded the rate threshold, measured as $(\mu+\sigma)$, were defined as an HFO area. It should be noted that the HFO rate is defined as the number of one-second windows that fulfill the energy requirement within the five-minute interval. For the rest of this paper, the HFO area refers to the sites identified by our analysis. 


\section{Experimental Results}

Two epilepsy patients, ID: Patient-11 and Patient-17, were considered in this study. Both patients underwent surgery to remove the SOZ. Seizure outcome of the resecting surgery was assessed in subsequent visits and classified according to the International League Against Epilepsy (ILAE). Patient-11 was reported seizure-free after surgery (ILAE Class 1, Table I); however, patient-17 still had recurrent seizures (ILAE Class 5, Table I). Fig. 2(a)(b) exhibit the anatomical position of the implanted electrodes in both patients along with the SOZ determined by clinicians, electrode sites with blue $\times$, and HFO areas obtained using our methodology as electrode contacts filled with red color. A total number of 19 and 1 fiveminute inerictal intervals were analyzed for HFO identification in the Patient-11 and Patient-17, respectively. Consistent with the notion that HFO generating areas are a suitable biomarker for the SOZ, our detected HFO channels in both patients well-overlap with the identified SOZ defined by clinicians, as illustrated in Fig. 2(a)-(b). All channels that were detected as HFO areas, in at least one of the analyzed intervals, for Patient-11 as well as a percentage for which those channels exhibit HFO activity are reported in Table II. The channels classified as HFO areas form a subset of the annotated SOZ. An interesting observation is that, in both patients, the HFO area is smaller than the annotated SOZ. Our analysis suggests the EZ in these patients might have been significantly smaller than the annotated SOZ identified by clinicians. In these patients, our HFO analysis could have significantly improved the surgical planning that defined the resected area.

Furthermore, we investigated the clinical relevance of the detected HFO areas. Patient-11, who was seizure-free after surgery, had his HFO area fully resected but not the entire SOZ. On the other hand, Patient-17 did not have a good seizure outcome despite the resection of most of the SOZ. Fig. 3 illustrates the area of the brain that surgically resected along with the annotated SOZ and the detected HFO area for Patient-17. In this patient, a large portion of the right frontal cortex was resected. While parts of the HFO areas, detected using our analysis, were included in the resection, we can see that the resection of the HFO areas was not complete. We believe our HFO analysis in concordance with the SOZ would have better delineated the EZ limiting the area of the brain required to be resected. This could have improved the seizure outcome for Patient- 17 with less destruction of brain tissue and thus fewer side effects.

\section{Conclusion}

In this study, an adaptive data-driven method is proposed to localize the EZ in patients with multi-channel iEEG. Empirical mode decomposition was utilized to extract a set of finite IMFs from bipolar iEEG signals. Hilbert transform of the IMFs was performed to measure their instantaneous frequencies. The energy of IMFs with frequencies lying in the ripple and fast-ripple bands along with rate thresholding were used to access the identification of the EZ. Testing on a preliminary dataset of two epilepsy patients has supported the feasibility of using our method to provide an automated algorithm that can be employed in concordance with the SOZ to better delineate the EZ. This could potentially lead to an improvement in the surgical planning of the resected area. The $\mathrm{H}$ Fo areas identified using our front-end analysis also appear to be clinically relevant. one patient where the HFo area was resected 
ended up seizure-free while the other patient had portions of the HFo area that were not resected and still had recurrent seizures. Although this protocol will need to be tested further over additional patients, it provides an analytical protocol that may have the advantages of maximizing surgical efficacy while minimizing neurological deficits that may arise as a result of the surgery.

\section{Acknowledgment}

The authors would like to thank the lab of Dr. Johannes Sarnthein at the university of Zurich, and colleagues at the Swiss Epilepsy Center, for providing us the clinical dataset and information regarding SoZs and resected areas.

*Research supported by National Institute of Health, United States, (R01 NS092760) to DJM.

\section{References}

[1]. Fisher RS, van Emde Boas W, Blume W, Eiger C, Genton P, Lee P, and Engel J, Jr., "Epileptic seizures and epilepsy: definitions proposed by the international league against epilepsy (ILAE) and the international bureau for epilepsy (IBE)," Epilepsia, vol.46, no. 4, pp. 470-472, 42005. [PubMed: 15816939]

[2]. Rosenow F, Lüders H, "Presurgical evaluation of epilepsy," Brain, vol. 124, no. 9, pp. 1683-1700, 9 2001. [PubMed: 11522572]

[3]. Staba RJ, Wilson CL, Bragin A, Fried I, and Engel J, Jr., "Quantitative analysis of high-frequency oscillations (80-500 Hz) recorded in human epileptic hippocampus and entorhinal cortex," J. Neurophysiol, vo 1.88, no. 4, pp. 1743-1752, 10 2002. [PubMed: 12364503]

[4]. Bragin A, Engel j., Jr., Wilson CL, Fried I, and Mathern GW, "Hippocampal and entorhinal cortex high-frequency oscillations $(100500 \mathrm{~Hz})$ in human epileptic brain and in kainic acid-treated rats with chronic seizures," Epilepsia, vol.40, no. 2, pp. 127-137, 8 1999. [PubMed: 9952257]

[5]. Jacobs J, Staba R, Asano E, Otsubo H, Wu JY, Zijlmans M, et al., "High-frequency oscillations (HFOs) in clinical epilepsy,” Prog. Neurobiol, vol.98, no. 3, pp. 302-315, 9 2012. [PubMed: 22480752]

[6]. Jacobs J, LeVan p., Chander R, Hall J,Dubeau F, and Gotman J, "Interictal high-frequency oscillations $(80-500 \mathrm{~Hz})$ are an indicator of seizure onset areas independent of spikes in the human epileptic brain,” Epilepsia, vol.49, no.11, pp. 1893-1907, 11 2008. [PubMed: 18479382]

[7]. Bragin A, Engel j., Jr., Wilson CL, Fried I, and Buzsaki G, "High-frequency oscillations in human brain,” Hippocampus, v o 1.9, no. 2, pp. 137-142, 4 1999. [PubMed: 10226774]

[8]. Jiruska P, Alvarado-Rojas C, Schevon CA, Staba R, Stacey W, Wendling F, et al., "Update on the mechanisms and roles of high-frequency oscillations in seizures and epileptic disorders," Epilepsia, vol.58, no. 8, pp. 1330-1339, 8 2017. [PubMed: 28681378]

[9]. Jrad N, Kachenoura A, Merlet I, Bartolomei F, Nica A, Biraben A, et al., "Automatic detection and classification of high-frequency oscillations in depth-EEG signals,” IEEE Trans. Biomed. Eng, vol. 64, no. 9, pp. 2230-2240, 9 2017. [PubMed: 28113293]

[10]. Fedele T, Burnos S, Boran E, Krayenbühl N, Hilfiker P, Grunwald T, et al., "Resection of high frequency oscillations predicts seizure outcome in the individual patient," Scientific Reports, vol. 7, no.1, pp. 13836-13846, 10 2017. [PubMed: 29062105]

[11]. Trongnetrpunya A, Nandi B, Kang D, Kocsis B, Schroeder CE and Ding M, “Assessing granger causality in electrophysiological data: removing the adverse effects of common signals via bipolar derivations,” Front. Syst. Neurosci, vol.9, no. 189, pp. 1-11, 12016.

[12]. Huang NE, Shen Z, Long SR, Wu MC, Shih HH, Zheng Q Yen N-C, and Liu HH, "The empirical mode decomposition and the Hilbert spectrum for nonlinear and non-stationary time series analysis," Proc. Royal Soc. London, vol. 454, no. 1971, pp. 903-995 31998.

[13]. Cho D, Min B, Kim J, and Lee B, "EEG-based prediction of epileptic seizures using phase synchronization elicited from noise-assisted multivariate empirical mode decomposition," IEEE Trans. Neural Syst Rehabil. Eng, vol.25, no. 8, pp. 1309-1318, 8 2017. [PubMed: 27775526] 
[14]. Sobayo T, Fine AS, Gunnar E, Kazlauskas C, Nicholls D, and Mogul DJ, "Synchrony dynamics across brain structures in limbic epilepsy vary between initiation and termination phases of seizures, IEEE Trans. Biomed. Eng, vol.60, no. 3, pp. 821-829, 3 2013. [PubMed: 22389141]

[15]. Gabor D, “Theory of communication”, J. Inst. Elect. Eng. London vol.93, no. 3, pp. 429-457, 11 1946. 


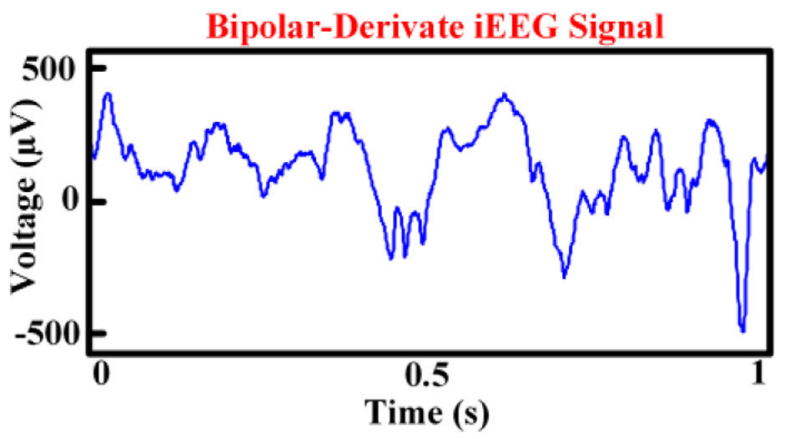

(a)

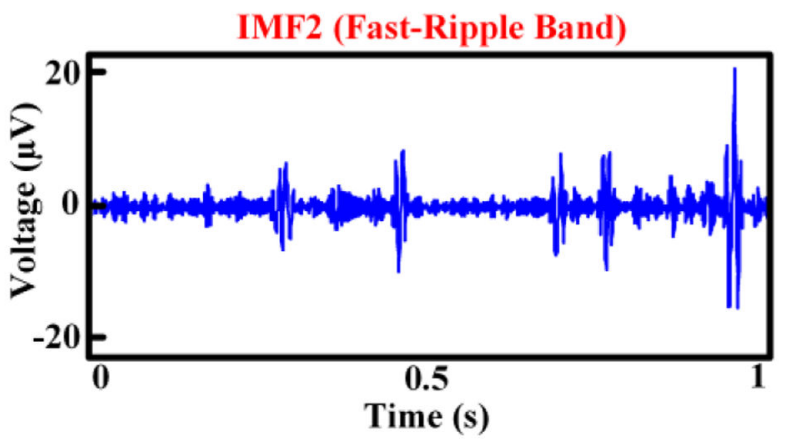

(b)

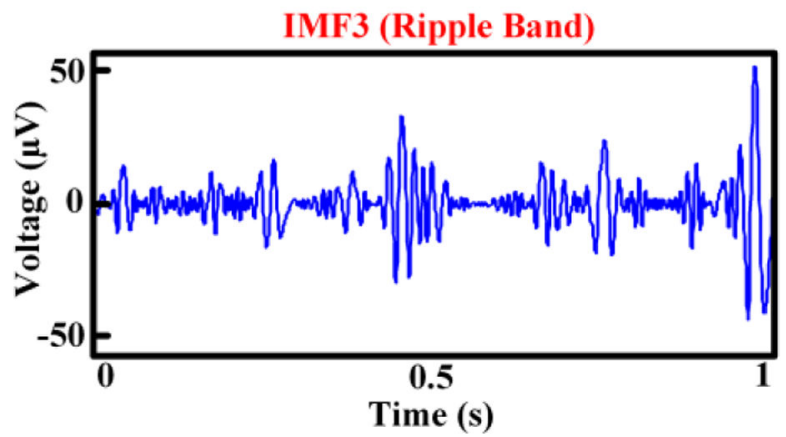

(c)

Fig. 1.

EMD process on a bipolar-derivate iEEG signal. (a) One-second, bipolar-derivate iEEG signal used for the EMD process. (b) The second IMF extracted from the iEEG signal, showing HFO activity in the fast-ripple band. (c) The third IMF extracted from the iEEG signal, showing HFO activity in the ripple band. 


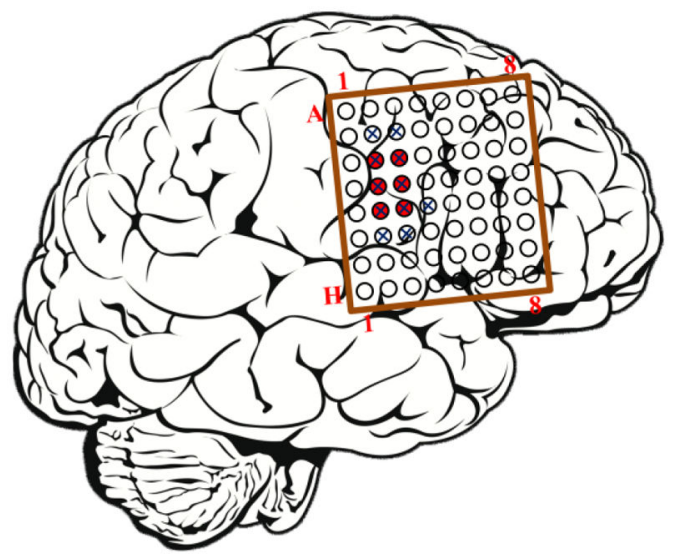

(a)

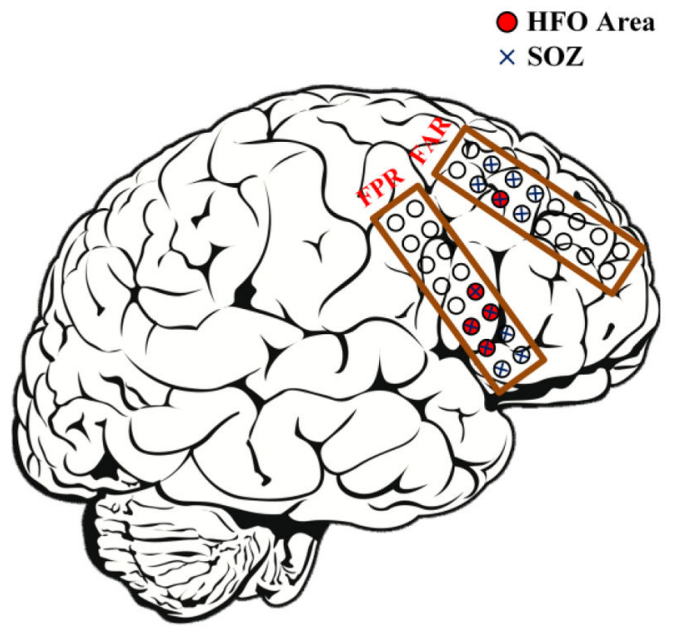

(b)

Fig. 2.

The HFO area detected in patients (Patient-11 and Patient-17) with extratemporal lobe epilepsy. (a) Anatomical position of the implanted grid-electrode for Patient-11.(b)

Anatomical position of the implanted grid-electrode for Patient-17. The SOZ identified by clinician are denoted as electrode contacts with the blue $\times$. The HFO area detected by our analysis is denoted by contacts filled with red color. 


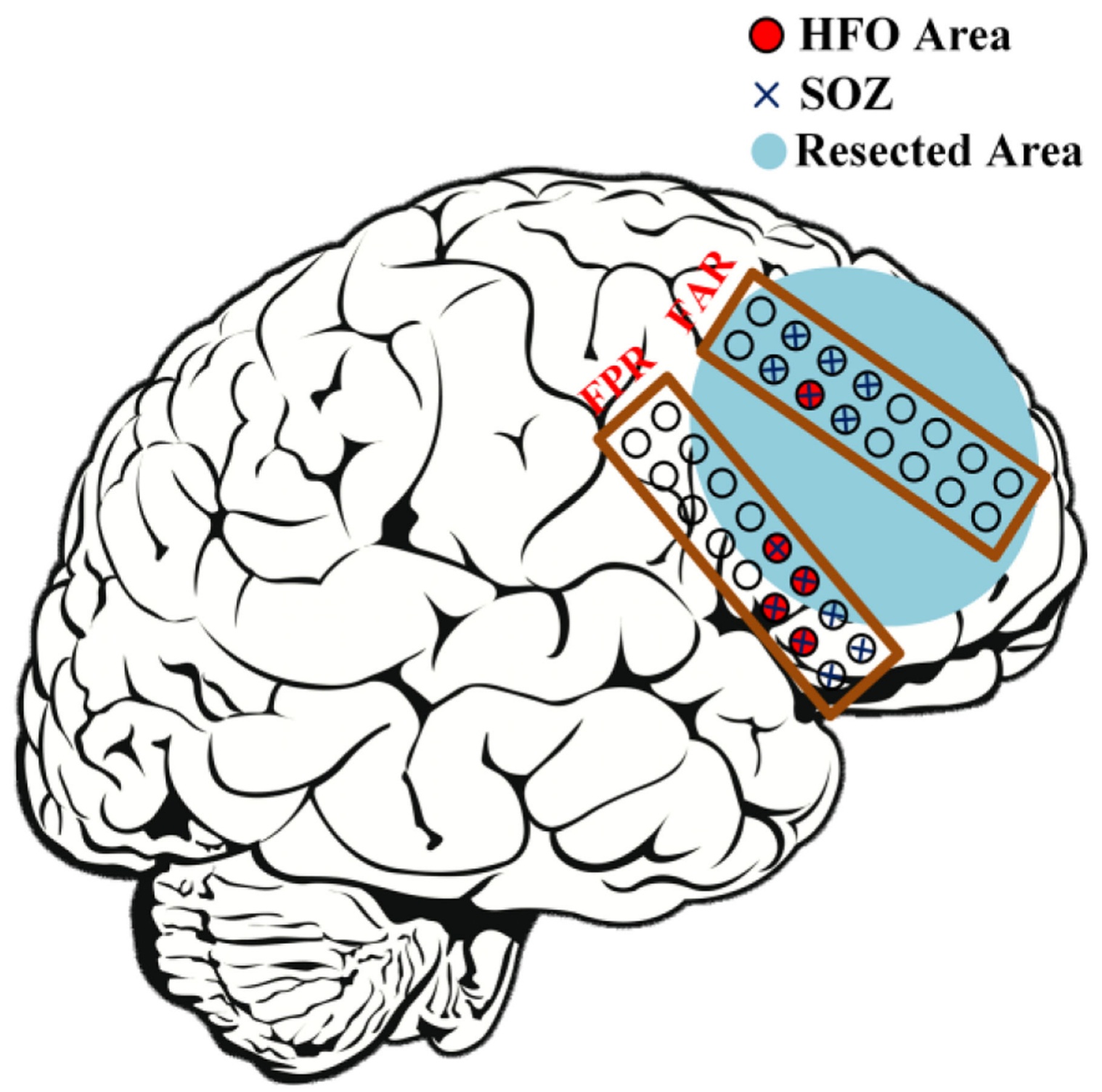

Fig. 3.

The HFO area exceeds the resected area in the patient with recurrent seizures (Patient-17). Two, out of the five electrode contacts displaying HFO activity, lie outside the resected area that may explain the patient's poor prognosis. 


\section{TABLE I.}

Patient Clinical Information

\begin{tabular}{c|c|c}
\hline Patient ID & Patient-11 & Patient-17 \\
\hline Age (y) / Sex & $36 / \mathrm{M}$ & $30 / \mathrm{M}$ \\
\hline Epilepsy & ETE & ETE \\
\hline Outcome (ILAE) & 1 & 5 \\
\hline $\begin{array}{c}\text { Types of } \\
\text { electrodes }\end{array}$ & $\begin{array}{c}1 \text { grid, } \\
8 \times 8\end{array}$ & $\begin{array}{c}2 \text { grids, } \\
8 \times 2\end{array}$ \\
\hline Nights & 3 & 1 \\
\hline Intervals & 19 & 1 \\
\hline
\end{tabular}

$\mathrm{M}=$ male; $\mathrm{ETE}=$ extratemporal lobe epilepsy; grid= grid electrodes; depth= depth electrodes 
TABLE II.

Channels Containing Hfo Activity In Patient-11

\begin{tabular}{c|c|c}
\hline $\begin{array}{c}\text { Channel } \\
\text { ID }\end{array}$ & $\begin{array}{c}\text { Num. of Intervals } \\
\text { with HFO activity }\end{array}$ & $\begin{array}{c}\text { Interval } \\
\text { Percentage * }\end{array}$ \\
\hline C2 & 2 & 11 \\
\hline C3 & 2 & 11 \\
\hline D2 & 9 & 47 \\
\hline D3 & 16 & 84 \\
\hline E2 & 14 & 74 \\
\hline E3 & 10 & 53 \\
\hline
\end{tabular}

*

represents percentage of intervals showing HFO activity out of the total of 19 recording intervals 